The BMJ

Cite this as: $B M / 2021 ; 373: n 990$ http://dx.doi.org/10.1136/bmj.n990 Published: 19 April 2021

\section{How can I succeed in a virtual consultant interview?}

The pandemic has increased the use of online meetings in a range of settings. Three consultant forensic psychiatrists with experience of conducting virtual interviews tell Abi Rimmer how you can succeed

Abi Rimmer

\section{Present yourself at your best}

Rebekah Bourne, consultant forensic psychiatrist, Birmingham and Solihull NHS Mental Health Foundation Trust, says, "How you present yourself is just as important in a video interview as in person, but making the right impression can be more challenging. How you dress, sit, and the setting are important in helping you to relax and showing yourself at your best.

"When choosing your outfit, make sure you are as smart as you would be in person. You may wish to make some slight adjustments to ensure you make an impact over the internet. Consider the colours you're wearing and the contrast with the background so you don't appear washed out. Some colours will show up differently to expected and patterns or stripes may create undesirable optical effects. Make sure you have a trial run to see how your outfit looks on camera.

"As with clothing, how you sit will be important. Most interviews last around an hour so you need to be comfortable so you appear relaxed and can use appropriate body language.

"There may be the opportunity to share your screen to illustrate your $\mathrm{CV}$ or publications. If this is a feature of your interview, make sure to do so in moderation so that you maintain rapport with the panel.

"While you have a choice of a natural or virtual background, I would favour the natural. If you do use a virtual background ensure it doesn't clash with your clothing and consider how the borders will appear with your natural body movements. If you are using your natural background, ensure it is appropriately 'staged' and professional and avoid over-personalisation.”

\section{Show your personality}

Michaela Routhu, consultant forensic psychiatrist, Southwest Centre for Forensic Mental Health Care in St Thomas, Canada, says, "At the moment your future employer might not actually meet you face-to-face until you start your job. To establish a rapport, I recommend using video calls where possible, including for the pre-interview meetings.

"Good visibility of your face is important in both virtual pre-interview meetings and interviews. Facial expressions are a crucial part of communication. Natural light is preferable but, depending on the time of the day or the weather, an artificial light may be needed. Ensure the direction of light is such that it does not blind the panel members in any way, or cause stark shadows on your face. Soft dispersed light usually works well. Give consideration to the distance from the camera, so that your face is visible enough.

"You might notice that you appear more tired in the video than in person. Make sure you up the energy levels as needed. Some visual information, such as body language and gestures, is lost during video calls. This puts more pressure on you to make sure your facial expressions represent what you would like to communicate.

"It's important to look into the camera lens, rather than at the picture of panel members, so that you don't appear to be looking down or to the side. This gives the impression of making eye contact. It might take some time to get used to this, but it's invaluable in engaging the panel members. Do, however, check the facial expressions of the panel from time to time for non-verbal feedback."

\section{Keep your composure}

Sunil Kumar Routhu, consultant forensic psychiatrist, Southwest Centre for Forensic Mental Health Care in St Thomas, Canada, says, "Many of us have seen the videos of interviewees being disturbed by unexpected visitors. In high stake interviews, such as consultant interviews, it's important to prevent such distractions.

"Find a space where you won't be disturbed. This doesn't necessarily need to be at home. The quieter the room, the better. Using earphones and a microphone will reduce background noises, any echoes from the computer, and limit distractions for all parties.

"Position yourself in a location with a strong internet signal. In the unfortunate event of a frozen screen, keep your composure. You can make a good impression by demonstrating your ability to deal with adversity. Even if the screen appears frozen, it's possible that the interview panel may still be able to see and hear you well. I advise continuing with the interview and checking with the panel if they want you to repeat yourself.

"The organisation will be able to give details of the software being used for the interview. Practise the virtual interview with a colleague, friend, or family member using the same software. The secretary or human resources staff from the organisation may even be able to arrange a short trial video call. If you're not sure, ask for help. Be aware that things could look different between computer, mobile, and tablet. 
"End the call in a professional manner. Wait for the panel members to disconnect before you leave the meeting room. To prevent mishaps, close the meeting window and terminate the audio and video connections like the microphone and webcam.” 